\title{
A Practical Exploration of Constructive English Learning Platform Informatization Based on RBF Algorithm
}

\author{
Ning Tang $\left(D,{ }^{1}\right.$ Bing Li, ${ }^{2}$ and Sang-Bing Tsai ${ }^{3}{ }^{3}$ \\ ${ }^{1}$ Basic Education Department, Henan Polytechnic, Zhengzhou, Henan 450018, China \\ ${ }^{2}$ College of Information Engineering, Henan Polytechnic, Zhengzhou, Henan 450018, China \\ ${ }^{3}$ Regional Green Economy Development Research Center, School of Business, Wuyi University, Nanping, China \\ Correspondence should be addressed to Ning Tang; tangning7980@163.com and Sang-Bing Tsai; sangbing@hotmail.com
}

Received 28 September 2021; Revised 27 October 2021; Accepted 1 November 2021; Published 18 November 2021

Academic Editor: Gengxin Sun

Copyright (c) 2021 Ning Tang et al. This is an open access article distributed under the Creative Commons Attribution License, which permits unrestricted use, distribution, and reproduction in any medium, provided the original work is properly cited.

\begin{abstract}
The constructive English learning approach provides better technical support and learning concepts for English learners. This paper firstly introduces the theoretical basis of constructive English learning and the status of domestic and international research and analyzes the advantages over the traditional English learning approach. This paper also analyzes the RBF neural network model, especially the locally adjusted RBF algorithm, and considers the training model and construction method of the RBF neural network to simulate the learning pattern of good learners of English in grades 4 and 6 to better realize the effect of the English learning platform and allow the English learning platform to play a supportive role in the learning process of students, and through the evaluation of the design, we can better improve the software design, which can be improved by evaluating the design. In the subsequent research, we should add a more user-friendly interface design, use larger model samples to train the model to make it more universal in accuracy and ensure good stability in operation, and integrate multiple algorithms to improve the training algorithm of RBF neural network to obtain a more general algorithm model. The rich teaching methods can cultivate students' independent inquiry ability, and the construction of this English learning platform can help improve students' ability to use information technology. By making students become the main subject of teaching and learning, we can stimulate students' enthusiasm and a good awareness of English learning. Using the radial basis function (RBF) algorithm and the constructive pod language teaching method, this English learning platform is a good and positive aid for students to learn English, and as a better English education tool, it has certain research value and positive significance for the study of English teaching.
\end{abstract}

\section{Introduction}

In recent years, with the rapid development of information technology, the Internet era has quietly arrived, bringing unprecedented convenience to people's lives. Communication tools such as smartphones, digital cameras, and computers have become an important part of life. At the same time, hundreds of millions of images, text, and video data are generated every day, and information is growing explosively. Because of the huge amount of data and the variety of formats, traditional data analysis tools and techniques are no longer able to handle this complex data [1] so that a sample in the test set can correspond to multiple sample labels at the same time. This chapter introduces the multilabel learning, RBF neural network, and ML-RBF multilabel learning algorithm. Therefore, the problem that needs to be solved now is how to extract valuable information from these huge and complex data to make the most beneficial decisions for users, and data mining technology is thus created. Data mining technology can extract valuable information and knowledge from large, messy, and incomplete, random, and vague application data [2]. With the development of the economy and increasing international exchanges, the ability to read, write, and communicate in English is very necessary. As the development of 
information technology can help students quickly improve their ability to master English, and students have higher and higher requirements for English teaching, the traditional English teaching model has been unable to meet the teaching needs [3]. The traditional English teaching model is no longer able to meet the teaching needs. Theoretical research on RBF-based neural network algorithms is used to explore the practice of constructive English learning platforms to immerse students in the English learning context, in addition to online teaching platforms widely used internationally, such as Blackboard, Moodle, WebCT, and eCollege.

The samples can belong to any of the tags and there is a correlation between each tag. In the clustering stage, not only $K$-means clustering is performed on the same tagged samples, but also $K$-means clustering is performed on all samples, and segmented basis functions are designed, and the above two clustering results are evaluated step by step in the new basis functions. Finally, the new basis function results are found. Compared with the traditional method, the correlation analysis is performed at the clustering stage based on the position of different sample centers to all sample centers, and the results of the two clusters are analyzed in the subsequent basis functions to find the correlation degree [4]. To analyze and master the application of traditional teaching methods in computer multimedia online teaching process (courseware management, resource management, self-study status tracking management, and personnel management), we carry out the platform's architecture design according to the three characteristics analyzed above and customize the software design standards of the relevant modules [5]. We developed and created the independent learning platform so that the platform can participate in all processes of English learning, develop personalized teaching programs, and obtain the best results in English learning in the shortest possible time for individual learning weaknesses. In addition, based on customer feedback, the software platform support equipment is improved and configured for adjustment.

In terms of theoretical significance, the step-by-step elements of microresource development and design are analyzed. Microlearning, with its simplicity, speed, and miniaturization, has played a positive role in improving the learning affect, motivation, and emotional experience. With the distance from the center point, it shows a monotonous decrease or a monotonous increase. As a linear function, the parameters of the radial function model: center, distance scale, and precise shape of the radial function are all fixed. The development and design of microlearning mainly focus on the development of microlearning platforms, the development of microlearning media, or the systematic design of microlearning methods. A complete system for the development and design of microlearning resources has not been formed yet. Therefore, this study aims to analyze a reasonable process of developing microlearning resources by combining the characteristics of microlearning resources themselves, the characteristics of microlearning, and the characteristics of the development disciplines. In terms of practical significance, the development and design of microlearning resources for college English courses is carried out; specific implementation methods and their rationality are proposed. A new school platform and a form of resources in line with textbook knowledge are created for college English courses, which meets the specific learning needs of learners, optimizes the learning experience of learners, and improves the effectiveness of learners' learning.

\section{Current Status of Research}

In the information age, the amount of text data and information in our lives is huge and growing rapidly. One of the important elements of data management tasks is how to manage textual data efficiently and quickly. In the process of text classification, a label set is given and then a classifier model is constructed based on the given sample data, which allows automated classification of text [6]. For example, a song can be classified as soothing or dynamic according to the rhythm, classical or country or other according to the content, or sad or positive according to the lyrics. Text classification has a wide range of uses today, and the emergence of digital libraries and various search tools has greatly reduced people's workload [7].

With the development of information science and technology and the diversification of information, multitag learning techniques have gradually matured and are widely used in automatic labeling techniques for biology, multimedia information, and entertainment items, such as music and movies [8]. In these fields, multitag learning algorithms mainly improved and optimized based on traditional classification algorithms. For example, a framework of a Bayesian-based multitag learning algorithm is proposed to better predict gene functions based on Bayes [9]. Deng et al. have done more in-depth research on multitag learning algorithms, outlining the basic concepts of multitag learning algorithms, evaluation metrics, and comparative experiments on several representative multitag learning algorithms [10]. After the study of multitag learning research results, the problem of how to make full use of the correlation between tags is the key to a deeper study of multitag learning algorithms, and researchers also tend to study this area [11]. Jie and Qiurui extended the MLKNN lazy learning algorithm to convert the multitag learning algorithm into multiple independent two classification problems [12]. However, this learning algorithm often fails to consider the correlation problem between labels, resulting in unsatisfactory classification results. Wang et al. made two different improvements based on the problems existing in MLKNN: first, for the test samples, the nearest neighbors consist of each subset of the label set, fully considering the information contained in the test samples [13]; second, in the prediction of label classes, the label count vector of Lin et al. proposed a multilabel Bayesian network algorithm considering the correlation between labels, and the effectiveness of the algorithm was fully demonstrated by the validation on the dataset [14]. In addition, Shanghai Normal University, University of Science and Technology of China, and other 
domestic universities, related faculty, and graduate students are also conducting multilabel research [15].

There are two main types of independent learning modes, including classroom independent learning and extracurricular independent learning, depending on the place of teaching. This type of learning mode requires students to complete their learning independently without external assistance. In contrast, independent learning in the classroom is often influenced by the teacher so that students can cooperate and get better learning results. In contrast, independent English learning is a process in which students plan and monitor their English learning according to their learning objectives to achieve good English learning results. The theoretical basis is based on constructivism, humanism, and cognitivism of modern educational psychology, which are based on the affective, cognitive, and environmental aspects of students' learning English to implement independent learning. The integration of these three theories provides the basis for a quality educational philosophy. In English learning activities, the constructive teaching model is mainly a student-led and teacher-led model that combines the two perspectives of teaching and learning, using advanced teaching concepts and combining information technology and multimedia technology to improve students' abilities in four aspects of English learning: listening, reading, writing, and listening. The object of constructive teaching design consists of three aspects: learning environment, learning activities, and media delivery.

\section{RBF Algorithm for Constructive English Learning Platform Informative Practice Design}

3.1. Analysis of the Improved RBF Algorithm. Multilabel learning refers to building a multilabel classifier model for a given sample dataset with labeled data so that a sample in the test set can correspond to multiple sample labels at the same time. This chapter introduces multilabel learning, RBF neural networks, and ML-RBF multilabel learning algorithms. For traditional machine learning, the purpose of a classifier is to label data with a label, and if a sample has two labels, then this is a binary classification problem [16]. The radial basis function model is a function that takes the Euclidean distance between the measured point and the sample point as an independent variable, uses the radial basis function as the kernel function, and uses linear superposition to form a model. A sample with multiple labels is a multiclassification problem. Multilabel learning is the learning process of labeling a sample with different labels. The difference between single-labeling and multilabeling is shown in Figure 1, where a sample with one label is a singlelabeling problem, and a sample with multiple labels is a multilabeling problem. See Figure 1.

The application of multitag learning is very promising. Previously, multitag learning was usually applied to text information classification and medical diagnosis. An article usually belongs to different categories, which may be sports, entertainment, or economics. A patient may also suffer from several different diseases, such as diabetes and hypertension. Recently, multitag learning has also been applied to more scenarios such as gene classification, automatic video annotation, and music classification. Each gene may have different kinds of markers for its function, such as metabolism and protein synthesis; in automatic video annotation, the content of each video may be associated with multiple things, such as pedestrians and buildings [17]. There are two main approaches to multitag learning: one is problem transformation and the other is algorithmic adaptation. The first main solution to the multitag learning algorithm is problem transformation; the main idea of the method is to convert the multitag learning problem into several already solved binary classification problems. This simplifies multitag learning by turning the more difficult models into several easy-to-implement models.

Radial functions are a special class of functions. The most typical characteristic of radial functions is that they are monotonically decreasing or monotonically increasing with distance from the center. As a linear function, the parameters of the radial function model: the center, the distance scale, and the exact shape of the radial function are all fixed. A typical radial function that we are familiar with is the Gaussian function, whose formula can be expressed as follows:

$$
h(x)=\exp \left(\frac{(x-c)^{2}}{r^{2}}\right) .
$$

Its parameters include the center $c$ and the radius $r$. The Gaussian radial basis function is characterized by a monotonically decreasing distance from the center. In contrast, the multiquadratic radial basis function is as follows:

$$
h(x)=\frac{r^{2}-(x-c)^{2}}{r^{2}} .
$$

The multiquadratic radial basis function is monotonically increasing with the distance from the center. The Gaussian radial basis function has a more pronounced local response characteristic, and the response is more significant in the neighboring region near the center, which is more commonly used than the global response of the multiquadratic radial basis function. The radial basis function model is a function of the Euclidean distance between the points to be measured and the example points as the independent variable, and the radial basis function as the kernel function, using a linear superposition to form the model. The radial basis function model can be roughly expressed as follows:

$$
f(x)=\sum_{i=1}^{m} \beta \varphi\left(\left\|x+x_{i}\right\|\right) .
$$

The RBF model can be constructed with very satisfactory fitting results according to both explicit and arbitrary correspondence. In addition, a comparative analysis based on individual kernel functions shows that satisfactory fitting results can be achieved in many scenarios by using Gaussian 


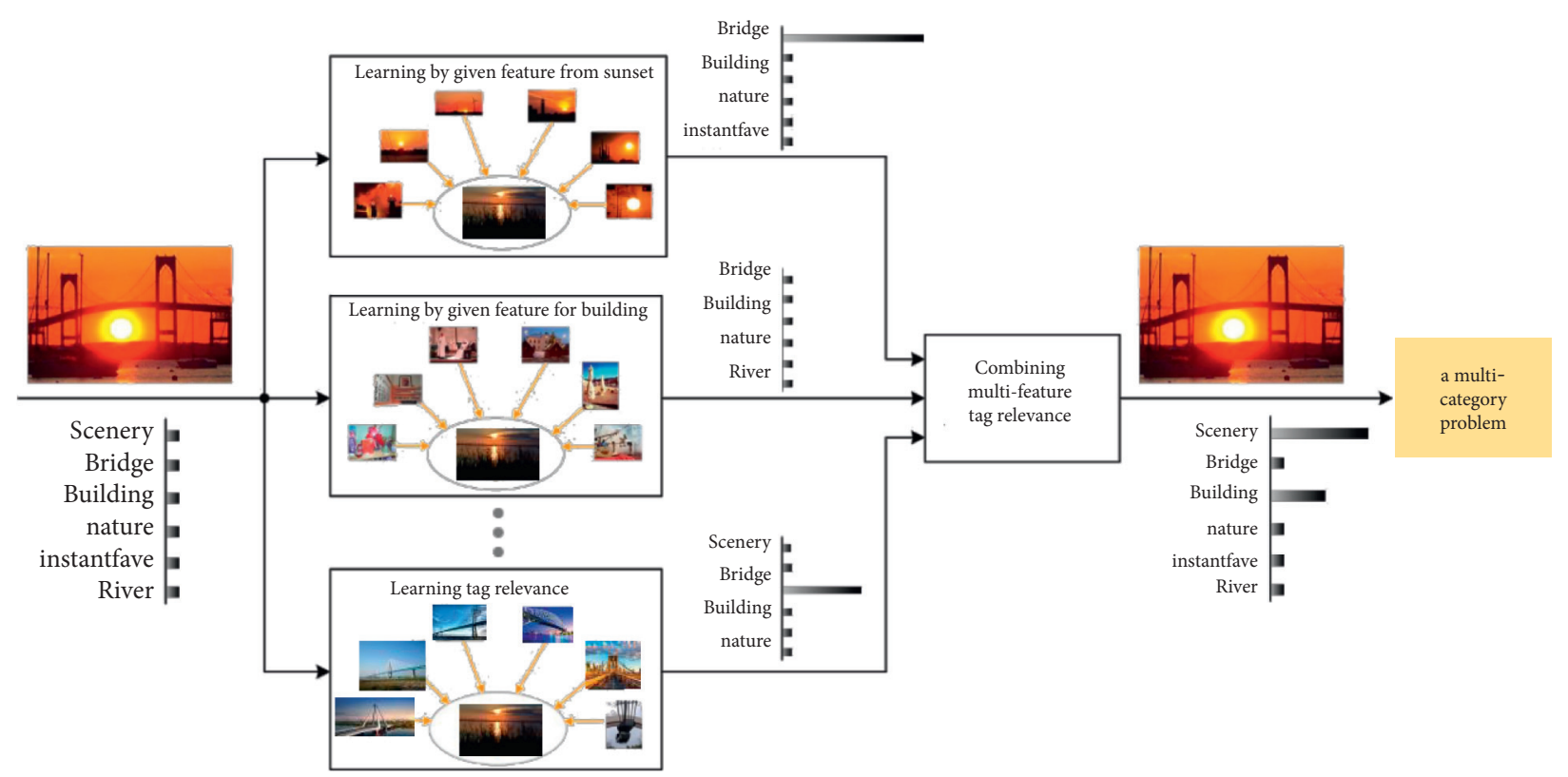

FIGURE 1: Single-tag versus multitag learning.

kernel functions as well as multiquadratic kernel functions. Therefore, in the present analysis, the online RBF learning algorithm is also formed by using Gaussian functions as kernel functions.

$$
\varphi(r)=\left(r^{2}-c^{2}\right)^{2}
$$

The model of constructive English learning platform based on RBF algorithm is mainly based on the idea of RBF algorithm in the design of constructive English learning platform, and the algorithm is fully reflected in the process of writing the code. The RBF algorithm can solve the quantitative indicators of English teaching, especially at the level of teachers, and how to optimize the teaching effect with different teaching times, teaching methods, and teaching content configuration. At the implementation level of the model algorithm, it can meet the needs of teachers and students. The RBF algorithm can solve the quantitative indicators of English teaching, especially at the teacher level, and how the teaching effect can be configured in different teaching time, teaching methods, and teaching content to achieve optimization. For students, the algorithm mainly addresses the learning performance under different learning states and task progress.

The algorithm model examines the performance of students in the national level 4 and 6 English exams. The input layer contains the study time of students under this learning platform, the implicit layer corresponds to the different contents of the study material, and the output layer is the performance of each subject, to calculate the optimal total performance of the level 4 and 6 English exams and to compare the proportion of time spent. To obtain the learning rule of good English proficiency, the data of first-year students and sophomores who took the level 4 and 6 English exams in some universities in a province where the first layer is the information factors related to the case; these inputs can be summarized as different English item indicators and input to the neural network structure. The second layer is the affiliation function, whose mathematical expression is as follows:

$$
\mu_{i j}\left(x_{i}\right)=\exp \left[\frac{\left(x_{i}^{2}+c_{i j}^{2}\right)^{2}}{\sigma_{i}^{2}}\right] .
$$

The point of algorithmic adaptation is to modify already existing single-label learning algorithms to enable them to solve multilabel learning problems. The basic idea of this approach is to improve traditional machine learning methods to solve multilabel problems. The underlying idea is that in the feature space, it is assumed that if the $k$-nearest neighbors around a sample have the same label, then that sample also has that label. It is worth noting that the KNN algorithm can be used not only for classification but also for regression. MLK is a lazy learning method that is improved from the KNN algorithm. For each unknown sample, the $k$ nearest neighbors in the training set are first determined. Then, the set of labels of the unknown samples is determined by computing the set of labels in its nearest neighbor samples, that is, the number of labels belonging to a specific class in the nearest neighbor examples, using the maximum a posteriori probability (MAP) principle. BRKNN is an improved KNN multilabel classification algorithm. Conceptually equivalent to combining the KNN algorithm and the popular Binary Relevance (BR) algorithm, additional changes are made to BRKNN according to the different features of the sample set, which improves not only the classification accuracy but also the processing speed. This is shown in Figure 2.

The radial basis function (RBF) space has gained widespread attention and rapid development in recent decades due to the advantages of storage and computation brought by its simple form, and the theory of RBF is well 


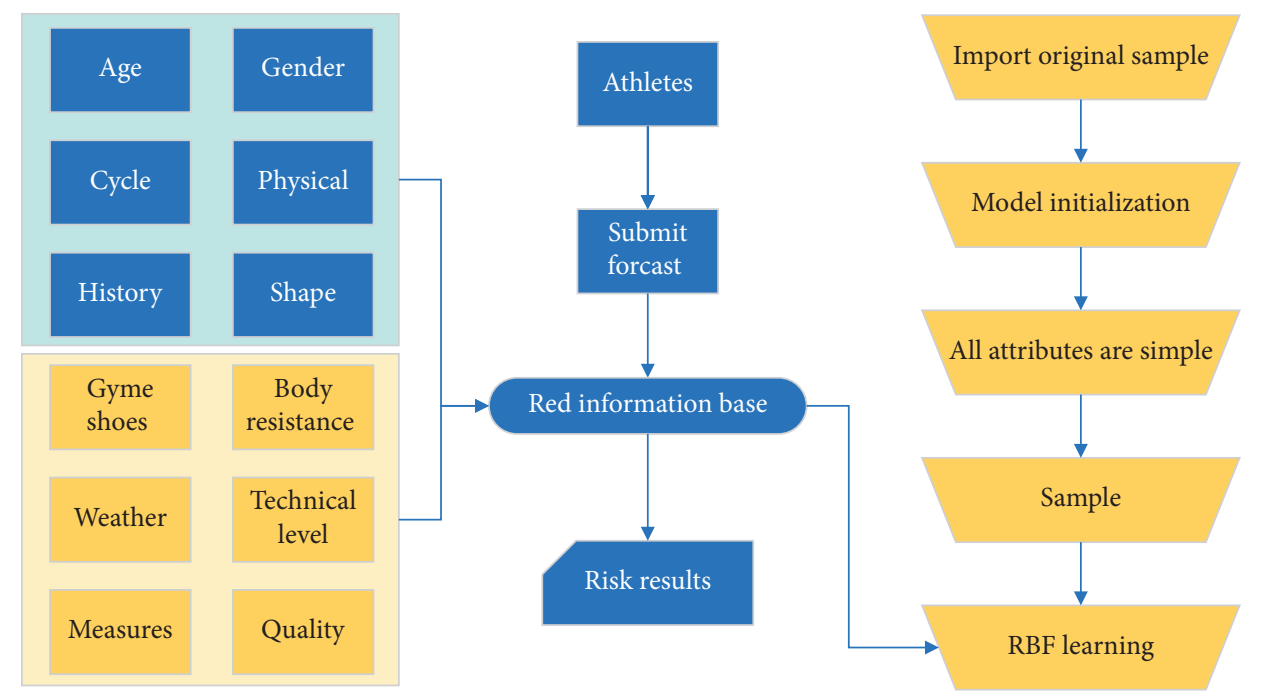

FIGURE 2: Structure diagram of the improved RBF algorithm neural network.

developed and widely used in various fields. However, most of the researches use radial basis functions as auxiliary functions of other machine learning tools, and the research on radial basis functions as independent data processing from the radial basis function itself is relatively thin. The radial basis function has a good development space in the field of time series prediction, and the online learning algorithm is also the current research hotspot. We can combine the radial basis function with big data and build the online learning algorithm based on a radial basis function for processing big data, which provides a new idea for the online learning field of big data. By calculating the label set in its nearby samples, that is, the number of labels belonging to a specific category in the nearby samples, the maximum posterior probability principle is used to determine the label set of the unknown sample.

Traditional learning algorithms for batch mode (i.e., offline learning algorithms) are premised on the assumption that the training data are known, and then a loss function is selected to solve the optimal model by processing the known samples. If one wants to find a more optimal model with a nonlinear function, the most common approach is to take kernel function learning. However, with the rapid development of cloud computing and IoT in recent years, the amount of data is getting larger and larger, and when the number of training samples reaches a certain scale, the difficulties faced when training either linear learning or kernel learning are very great. Both in terms of computational volume, processing difficulty, and even accuracy will be greatly reduced. It is the difficulty of offline learning that has accelerated the development of incremental learning algorithms. This is because incremental learning algorithms can solve these difficulties very well. Incremental learning assumes that the training data can come sequentially. At each moment, the training data receives a brand-new sample, and we can decide whether to update our model by receiving this new training sample based on whether the performance of the model constructed from the previous training sample meets our experimental requirements.
Compared with the traditional offline learning, the incremental learning model update only needs to use the new samples received at this moment based on the previous model, which greatly reduces the computational complexity and time cost. Even if all training samples are obtained at once, incremental learning can still be used to train additional samples based on the previous training, reducing the complexity of training, reducing workload, and saving time costs.

$$
A=\left[\begin{array}{cccc}
\varphi\left(\left\|x+x_{1}\right\|\right) & \varphi\left(\left\|x+x_{2}\right\|\right) & \ldots & \varphi\left(\left\|x+x_{t}\right\|\right) \\
\varphi\left(\left\|x+x_{2}\right\|\right) & \ldots & \ldots & \varphi\left(\left\|x+x_{t+1}\right\|\right) \\
\varphi\left(\left\|x+x_{3}\right\|\right) & \ldots & \ldots & \varphi\left(\left\|x+x_{t+2}\right\|\right) \\
\varphi\left(\left\|x+x_{4}\right\|\right) & \ldots & \ldots & \varphi\left(\left\|x+x_{t+3}\right\|\right)
\end{array}\right]
$$

The output expression of the radial basis function is

$$
f(x)=\sum_{i=1}^{t} \beta_{i} \varphi\left(\left\|x_{1}+x_{t}\right\|\right) .
$$

From equation (7), the inverse function A1 of the radial basis kernel function A must be found. In the case of the high determinant dimension of $\mathrm{A}$, the complexity of finding the inverse is larger, so it can be solved by the technique of block matrix calculation.

3.2. English Learning Information Platform Design. To facilitate the reader's understanding of the overall operation logic, the platform is only based on the business related to the platform; from the overall technical architecture, the specific architecture is shown in Figure 3 below.

Using a distributed network topology, the platform supports different identity rights according to the user levels of teachers, students, and administrators, while the corresponding services are slightly different. 


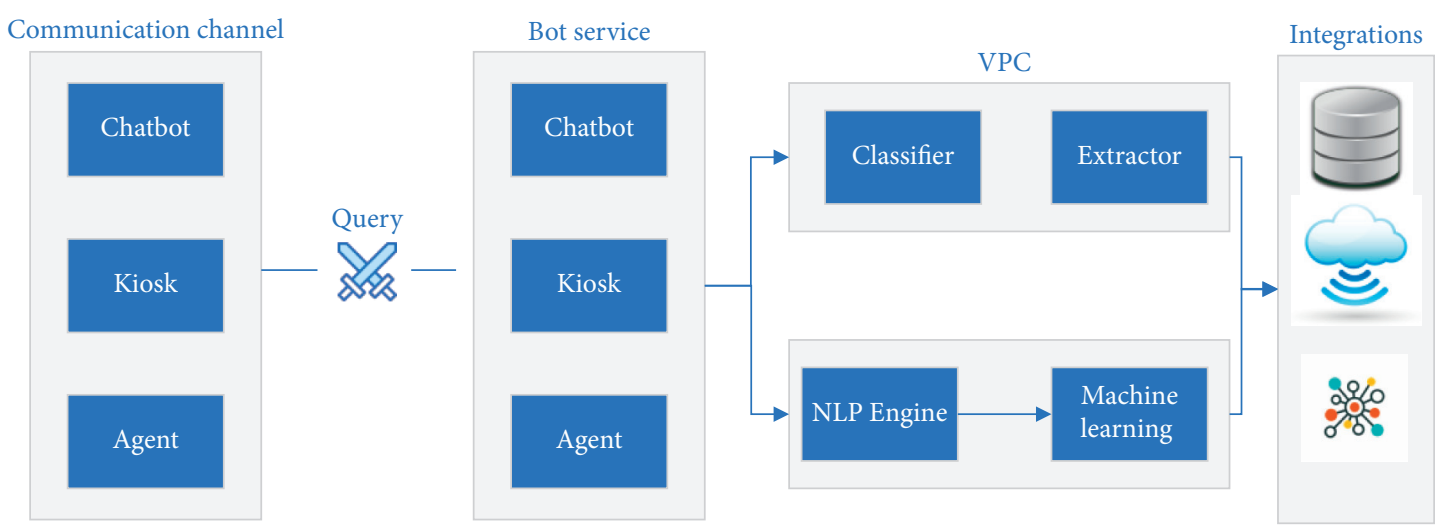

FIGURE 3: Logical interaction architecture diagram.

The package structure in the Provider can be adjusted according to your needs. The logical processing layer interacts with the backend data layer using Redis as a cache, and the data in Redis is read first, and if the data is not stored in Redis, Redis is refreshed first [18]. The logical processing layer contains two important business contents, which are also the focus of this thesis, namely, teacher recommendation and video streaming analysis. The teacher recommendation business receives information from the presentation layer, such as student information, teacher information, teacher matching $\log$, and teacher-student evaluation log. The teacher recommendation business uses Spark to perform offline computation on this information, and the computation information during the algorithm is stored persistently in the HDFS file system using the HDFS Connector [19-22]. At the same time, when the teacher recommendation list request is sent by the teacher viewing business, Spark streaming is responsible for real-time computation of the data in Kafka, and the computation results are directly stored in MySQL. The related theories of the radial basis function are also well developed, and they are widely used in various fields. However, most studies use it as an auxiliary function for other machine learning tools, starting from the radial basis function itself. Research on independent processing of data is relatively thin. The teacher recommendation list corresponding to the current student is fed back to the teacher viewing business, and the teacher viewing business returns the teacher information to the presentation layer.

The platform implemented based on modern computer network communication technology and multimedia technology. The platform closely combines teachers, students, and teaching resources, applies intelligent methods such as teaching methods and learning skills assessment to the teaching management process, achieves organic integration management of various subjects (teachers, students, courseware, and aids), teaching modes (organization, management, assessment, evaluation, and strategies), teaching behaviors, shows students' personalized learning and teachers' unique teaching, and provides an assessment of teachers' teaching skills. A strong basis for teachers' course courseware organization is as shown in Figure 4.
For vocabulary, the goal is to add approximately 2,000 English words to your high school vocabulary. The goal of improvement is to be able to communicate independently for your future work and professional life by adding about 3000 English words to your high school vocabulary and being able to communicate formally in writing on topics you are familiar with. The developmental goal is to be able to communicate independently and effectively for future work and study and to be able to use their knowledge of English to understand effectively and express themselves independently in writing or orally in English materials about their profession that are difficult to understand. At the same time, based on the three teaching macro-objectives, we combine the characteristics of the English subject [23-26]. They are divided into three categories: listening comprehension, oral expression, reading comprehension, written expression, and translation. The current research object learners were selected from the second-year students of primary education in a university in Anhui Province. The basic level teaching objectives are set for students who passed the English Advanced Level Examination, which is the requirement that most students should achieve by the time they graduate from the undergraduate program. The improvement goals and development goals aimed at students who have passed the English entrance examination at the university entrance, which is the goal proposed for majors with higher requirements for English application skills.

The live course implementation closely related to thirdparty services grouped into a module fast, because, for student users, the class is just a process of generating course playback with the teacher Taoshonai, etc. Meanwhile, the process of building a live classroom, obtaining classroom information, entering the classroom, and generating course playback in the live broadcast, as well as the pushing stream of video audio and video, consistent sound, and pictures, and lagging during the live broadcast, are also interactive live course modules required to solve the problem.

The courses of this learning platform are prepared by professional teachers, through scientific research on English teaching into levels, units, and courses, with multiple units under each level, and multiple under each unit, students can only enter the next unit after taking all the courses under a unit in order, and after taking all the units of the current 


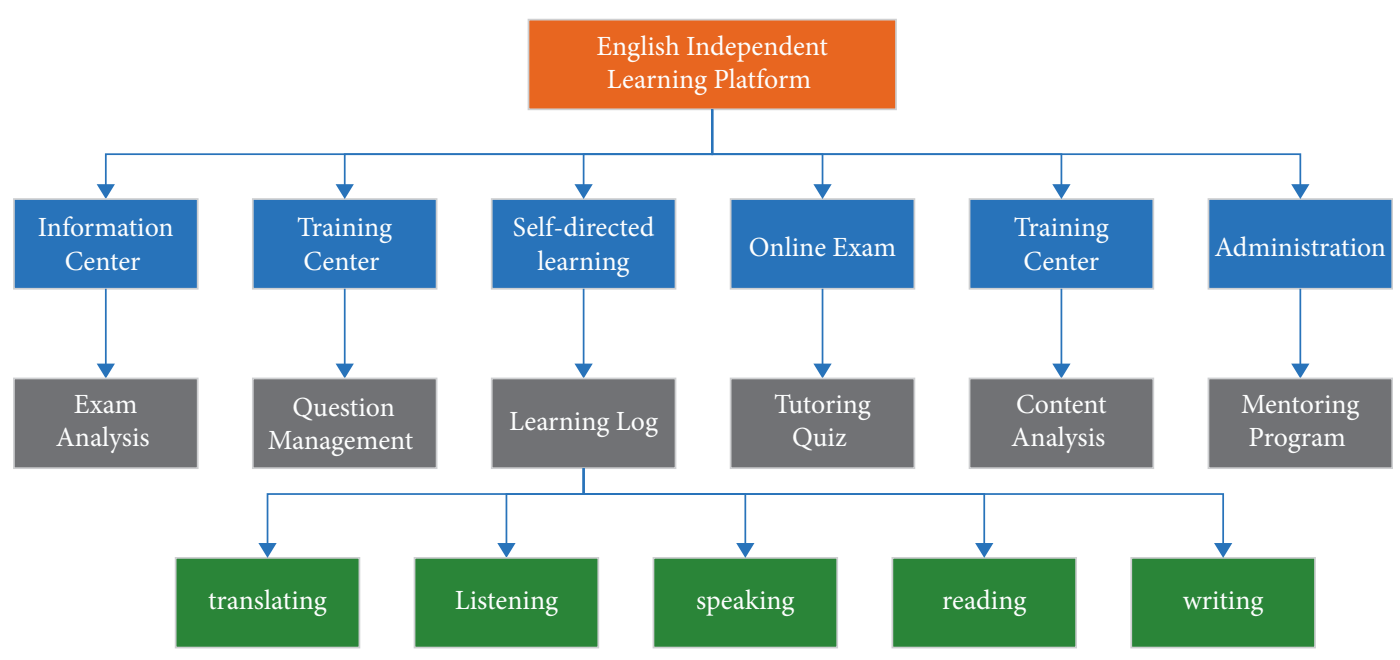

Figure 4: System functional structure diagram.

level, they can enter the next level for learning, the platform through the way of course tree. The platform stores courses utilizing a course tree. The current problem that needs to be solved is how to extract valuable information from these huge and complex data and make the most beneficial decision for users. Data mining technology is born from this. The platform has designed corresponding exercises for each lesson as postlesson assignments for students to test and verify their learning. After a new student pays the membership fee, he/she must take a professional English level test to determine his/her English level, and then he/she can proceed to the lessons corresponding to his/her current level, and before each lesson, he/she only needs to make an appointment with the teacher and take the lessons in the order prepared by the platform. The student entity can take multiple English tests, and each time a test record will be generated to record the current level of the student.

\section{Analysis of Results}

4.1. Results of RBF Incremental Learning Algorithm. The radial basis function-based windowed online learning algorithm model is the model constructed with a medium window length of 500, which is used to train the remaining data in the dataset. The online learning algorithm uses a sliding window of constant length, and each time a new data sample arrives, an old data sample is removed from the sliding window, thus ensuring that the length of the sliding window remains constant, and all datasets are processed sequentially in the order of their arrival. Since one old piece of data is deleted each time new data arrives, the total number of samples processed each time is fixed, so the time required to update the model each time is not very long, which greatly reduces the complexity of building the model and effectively solves the problem of dynamic streaming data processing. Since this experiment is conducted in two parts, the dataset is divided into two parts: the first part is the data used for incremental learning, and the first 500 data are taken for the experiment; the second part is the data of the windowed online learning algorithm for streaming dynamic data, and the last 3453 data of the dataset are taken for the experiment.

The implementation and simulation of the radial basis function-based learning algorithm for this experiment are based on the MATLAB implementation. The experimental results of incremental learning, as well as offline learning, are shown in Figure 5. To ensure the fairness of the experiments, the incremental algorithm and the offline algorithm of the above experiments are conducted in the same environment of the experiments and the same set of datasets are used. The time duration of incremental learning is much shorter than that of offline learning for the same sample set. It indicates that the incremental learning algorithm effectively solves the problem of long computing time for massive data. The real-time interaction process between users and customers generates a large amount of data, and the incremental algorithm not only makes up for the lack of machine storage space but also improves the training efficiency of data processing and greatly reduces the time cost of data processing. See Figure 5.

Moreover, students have put forward higher and higher requirements for English teaching. The traditional English teaching model has been unable to meet the teaching needs. Scholars at home and abroad are exploring autonomous constructive English teaching methods and using information technology to teach this kind of teaching. After the experiment with the first 500 data in the dataset, the first 500 data are used as the initial data to build an online RBF model, and the last 3453 data in the dataset are processed online in real time. The window of this experiment is fixed at 500, and then each time a new data is incremented, an old data in the window is deleted, the model is updated, and then the next data is predicted, and so on, until the data training is finished and the experiment is completed. The error results of this experiment on streaming data processing are shown in Figure 6.

Simple statistical processing of the dataset shows that the basic situation of the variable "average volume in the last 30 days" (i.e., the volume of sales to be predicted in this experiment) among the 18 variables is as follows, where the minimum value is 18 , the median is 56 , the average is 218.3 , and 


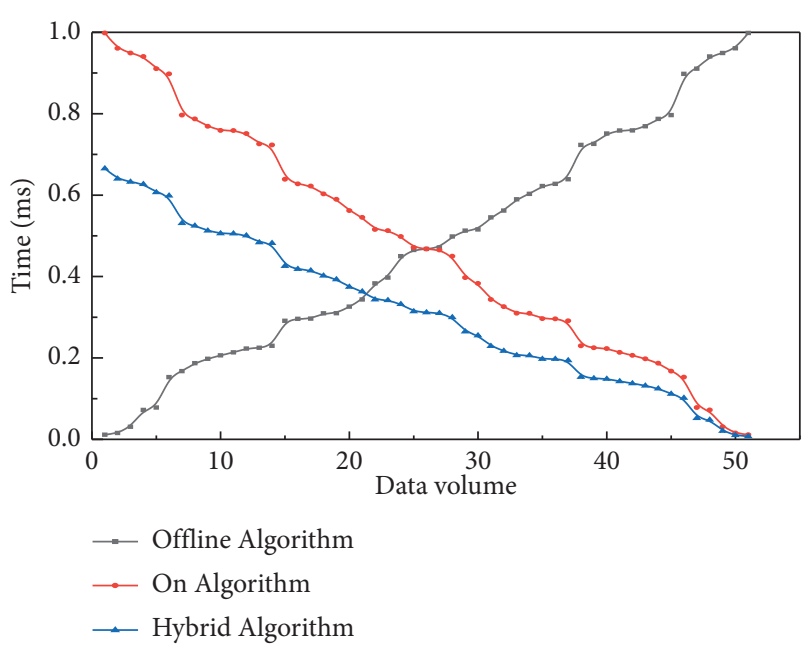

FIgURE 5: Time spent on an incremental learning line graph.

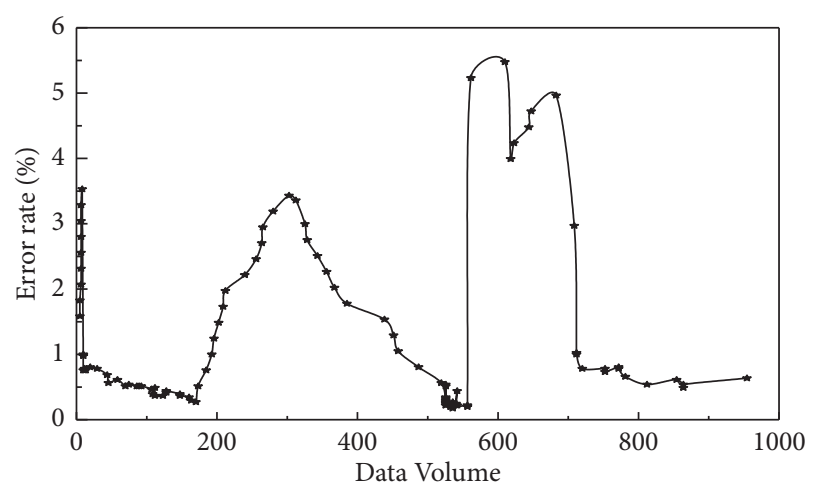

FIGURE 6: Error result curve of online RBF algorithm.

the maximum is 9798 . The error results for the latter 3453 data in the dataset are shown in the above graph, and the average error rate for these 3453 error results is found to be $0.2251 \%$. Both the magnitude of the difference in the standard error and the experimental result of the average error rate are relatively accurate and well processed in real-time for the streaming data, which validates the effectiveness of the online RBF learning algorithm. The large fluctuation in Figure 6 to the left of the horizontal coordinate of 2500 is because the store took some marketing measures to promote the activity at a lower price than usual during the time of that sales volume, which prompted a large gap between the sales volume and the usual sales volume, thus leading to the experimental results. However, the overall prediction result of this experiment is relatively flat and still convincing. The algorithm adopted in this experiment is suitable for more conventional sales prediction, and the fluctuations may be larger for the prediction of unexpected states and other episodic situations, but the experimental errors are within the acceptable range, and the online RBF learning algorithm is still effective for handling streaming dynamic data.

4.2. Performance Results of the Constructive English Informatics Platform. On the software side, the use of mute technology
148 and time-based control 91 video playback allows a video to be reused in multiple forms in the system, which is suitable for personalized learning and reduces the space occupied by resources. The software can transmit data normally without being affected when the bandwidth is very small, and it can pass through different security system interceptions, which can adapt to the learning and data transmission in the current state of the intricate network environment. The client-side can interact with the resources and the server side can receive the data submitted for online learning behavior. In terms of hardware resources, high-end compression technology is used to achieve lossless compression of various data, including audio-visual data and information data, which saves the investment cost of hardware resources. And design a piecewise basis function, and evaluate the above two clustering results step by step in the new basis function. Finally, find the new basis function result.

For language learning, the system has stable and powerful technical support for students to train in listening, reading, and writing. Language training can have features including adjusting the listening speed level, adjusting the reading speed, comparing with reading training, recording assessment, and role-playing of students' participation in language situations. The overall interaction technology of the software achieves simplicity and uniformity, which includes mouse operation, icon buttons, and playback controls. The demonstration application unit of the overall platform in this paper is a local comprehensive university in Hubei, which is formed by the merger of six local colleges, with more than 34,000 students and teachers, and a relative shortage of teachers. The ratio of English teachers to students has reached $1: 150$ to $1: 180$, which cannot guarantee the teaching effect, plus the lack of modern teaching equipment; students have little opportunity to use multimedia and speech equipment. The university English is a universitywide basic course, and teachers are generally burdened with heavy teaching tasks and have no time for further training and research thinking, lacking innovative teaching concepts and innovative spirit, as shown in Figure 7.

The mobile learning platform built on the WeChat public platform has the advantage of real-time and accurate pushing of learning resources. However, the data from the background shows that the number of learners reading the learning resources fluctuates greatly. At the beginning of the push, the number of readers of a learning resource was only a few, and the number of readers grew gradually only after continuous real-time communication with learners and continuous adjustment of the size and format of the learning resources. However, as time went on, the interest of learners seemed to decrease. The number of people reading the resources tended to decrease again, and the overall trend fluctuated significantly. This indicates that the effectiveness of the resources in the process of pushing learning resources is unstable, the learners' self-control is not very strong, and it is difficult to discipline themselves for a long time, and their motivation to learn is insufficient at the later stage.

Testing is the foundation of functional and quality assurance for a software product and is a process that must be 


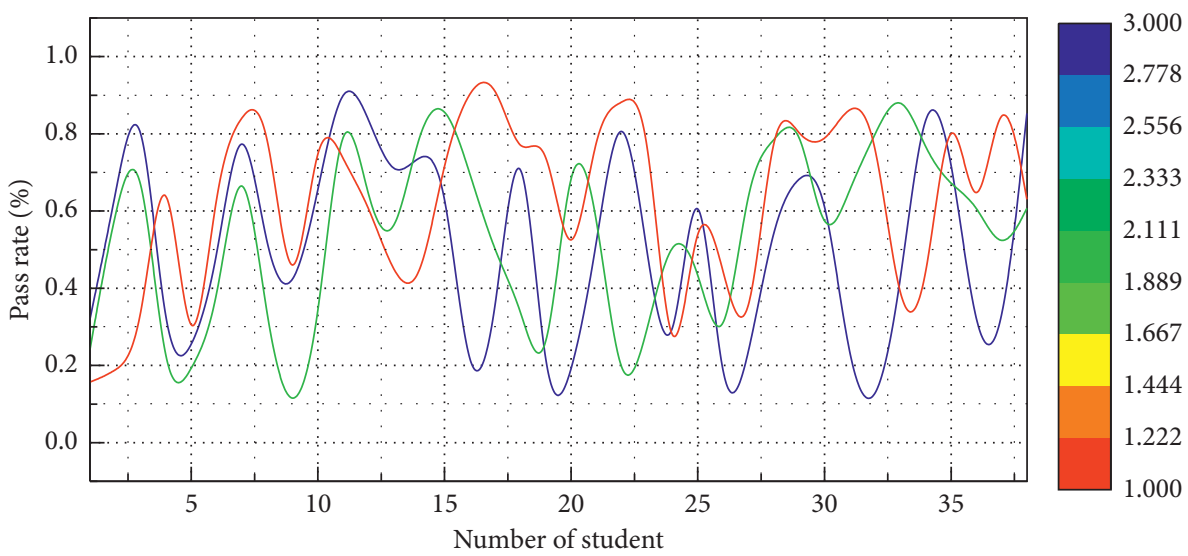

Figure 7: Exam results.

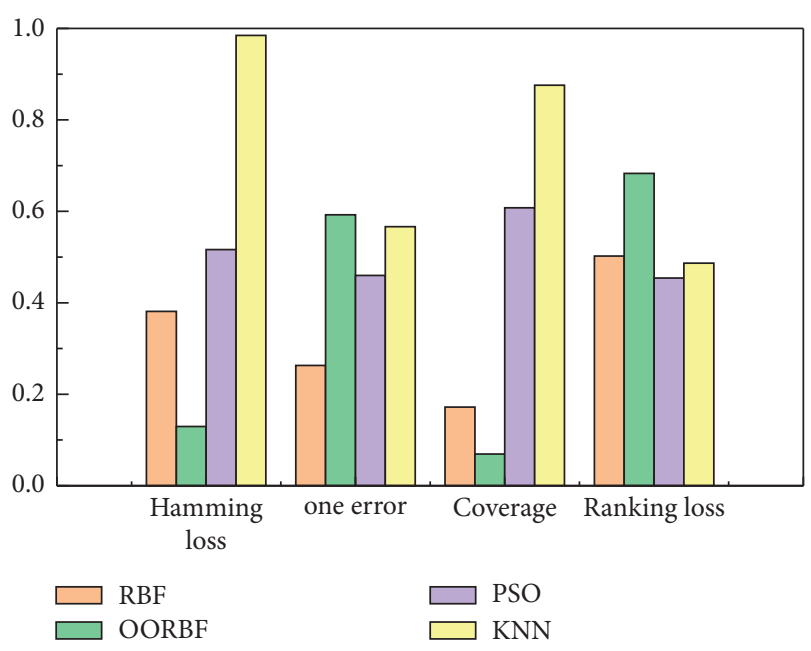

Figure 8: Performance comparison.

undergone before going live. In this process, the whole platform is taken as a whole, and the feedback and performance behavior of this whole is searched for in the case of external inputs and interventions to ensure the correct execution of functional processes so that errors implied in the analysis and design implementation process can be found and corrected before being used by users. And the design of this testing process is divided into functional and nonfunctional testing. Functional testing is mainly to experience the use of each functional module of the platform to check whether the product functions can meet the original design and user requirements; nonfunctional testing focuses on checking the quality of the product from the perspective of the stability and security of the platform itself, as shown in Figure 8.

We analyze the research and application status of online English self-learning platforms at home and abroad and analyzed the application of constructive teaching model in the English teaching process (learner testing, assessing learners, arranging teaching plan, learner self-study, completing self-study assignments, and retesting and assessing process). We analyzed the application of traditional teaching methods in the process of computer multimedia network teaching (resource management, courseware management, personnel management, and self-study status tracking management), the architectural design of the platform according to the characteristics analyzed above, and the development of software design specifications for relevant modules.

\section{Conclusion}

In the big data-oriented environment, this paper conducts in-depth research around the basic theories of radial basis function, incremental online learning, and windowed online learning and proposes incremental learning algorithms based on radial basis function for big data according to the process of incremental learning. For the high-dimensional feature space of data streams, it is difficult to solve the selection of many features. In this paper, the computational principle of block matrix inversion and the mechanism of incremental with sliding indefinite long window is used to reduce the complexity of processing big data, improve the computing efficiency of big data processing, and improve the generalization performance of the algorithm to simplify the complex big data learning process. We developed and designed microlearning resources for college English courses, proposed specific implementation methods and their rationality, and created a new school platform and textbook knowledge for college English courses to meet the needs of learners. Specific learning needs optimizing the learning experience of learners and improving their learning effectiveness. Incremental big data learning algorithm based on radial basis function, research based on the advantages of radial basis function itself space storage and computing power, and design with adjustable sliding window after can have the ability of big data incremental learning, and after experiments, it is confirmed that the big data incremental learning algorithm can effectively deal with big data. We have developed and implemented an independent learning platform that allows the platform to participate in the whole process of English learning, develop personalized teaching plans, and achieve the best results in English learning in the shortest time for individual learning weaknesses. At the same time, according to customer feedback, the software 
platform support equipment to adjust and improve the configuration.

\section{Data Availability}

The data used to support the findings of this study are included within the article.

\section{Conflicts of Interest}

No conflicts of interest exist concerning the publication of this study.

\section{References}

[1] D. Li, L. Deng, and Z. Cai, "Statistical analysis of tourist flow in tourist spots based on big data platform and DA-HKRVM algorithms," Personal and Ubiquitous Computing, vol. 24, no. 1, pp. 87-101, 2020.

[2] H. Jiang, K. Chen, Q. Ge, Y. Wang, J. Xu, and C. Li, "Fault diagnosis of power IoT system based on improved Q-KPCA$\mathrm{RF}$ using message data," IEEE Internet of Things Journal, vol. 8, no. 11, pp. 9450-9459, 2021.

[3] S. Usharani, D. Jayakumar, D. U. Palani et al., "Industrialized service innovation platform based on $5 \mathrm{~g}$ network and machine learning," European Journal of Molecular \& Clinical Medicine, vol. 7, no. 11, pp. 5684-5703, 2020.

[4] L. Yang, J. Luo, J. Luo, Z. Wang, Y. Chen, and C. Wu, "Research on recognition for cotton spider mites' damage level based on deep learning," International Journal of Agricultural and Biological Engineering, vol. 12, no. 6, pp. 129-134, 2019.

[5] Z. Yang, W. Li, W. Li, M. Li, and X. Yang, "Automatic greenhouse pest recognition based on multiple color space features," International Journal of Agricultural and Biological Engineering, vol. 14, no. 2, pp. 188-195, 2021.

[6] L. Li, P. Jiang, H. Xu, G. Lin, D. Guo, and H. Wu, "Water quality prediction based on recurrent neural network and improved evidence theory: a case study of Qiantang River, China," Environmental Science and Pollution Research, vol. 26, no. 19, pp. 19879-19896, 2019.

[7] Q. Fu, W. Shen, W. Shen et al., "Predicting the excretion of feces, urine and nitrogen using support vector regression: a case study with Holstein dry cows," International Journal of Agricultural and Biological Engineering, vol. 13, no. 2, pp. 48-56, 2020.

[8] L. Ting, J. Yuhan, Z. Man, S. Sha, and L. Minzan, "Universality of an improved photosynthesis prediction model based on PSO-SVM at all growth stages of tomato," International Journal of Agricultural and Biological Engineering, vol. 10, no. 2, pp. 63-73, 2017.

[9] J. Chen, X. Zhuo, F. Xu, J. Wang, D. Zhang, and L. Zhang, “A novel multi-classifier based on a density-dependent quantized binary tree LSSVM and the logistic global whale optimization algorithm," Applied Intelligence, vol. 50, no. 11, pp. 3808-3821, 2020.

[10] X. Deng, P. Jiang, X. Peng, and C. Mi, “An intelligent outlier detection method with one class support tucker machine and genetic algorithm toward big sensor data in internet of things," IEEE Transactions on Industrial Electronics, vol. 66, no. 6, pp. 4672-4683, 2018.

[11] M. M. Ali, "Arabic sentiment analysis about online learning to mitigate covid-19," Journal of Intelligent Systems, vol. 30, no. 1, pp. 524-540, 2021.
[12] Z. Jie and M. Qiurui, "Establishing a Genetic Algorithm-Back Propagation model to predict the pressure of girdles and to determine the model function," Textile Research Journal, vol. 90, no. 21-22, pp. 2564-2578, 2020.

[13] X. Wang, Y. Chai, H. Li, W. Wang, and W. Sun, "Graph convolutional network-based model for incident-related congestion prediction: a case study of Shanghai expressways," ACM Transactions on Management Information Systems, vol. 12, no. 3, pp. 1-22, 2021.

[14] Z. Lin, J. Jia, W. Gao, and F. Huang, "A hierarchical coarse-tofine perception for small-target categorization of butterflies under complex backgrounds," Journal of Intelligent \& Fuzzy Systems, vol. 38, no. 3, pp. 3463-3487, 2020.

[15] J. Yin, X. Liu, X. Liu et al., "Measurement and prediction of tomato canopy apparent photosynthetic rate," International Journal of Agricultural and Biological Engineering, vol. 12, no. 5, pp. 156-161, 2019.

[16] M. Kang, X.-R. Fan, J. Hua, H. Wang, X. Wang, and F.-Y. Wang, "Managing traditional solar greenhouse with CPSS: a just-for-fit philosophy," IEEE Transactions on cybernetics, vol. 48, no. 12, pp. 3371-3380, 2018.

[17] X.-J. Wang, G.-T. Zeng, K.-X. Zhang, H.-B. Chu, and Z.-S. Chen, "Urban real estate market early warning based on support vector machine: a case study of Beijing," International Journal of Computational Intelligence Systems, vol. 13, no. 1, pp. 153-166, 2020.

[18] D. Li, Z. Cai, L. Deng, and X. Yao, "IoT complex communication architecture for smart cities based on soft computing models," Soft Computing, vol. 23, no. 8, pp. 2799-2812, 2019.

[19] C. Li, Y. Zhang, G. Zhao, and Y. Ren, "Hourly solar irradiance prediction using deep BiLSTM network," Earth Science Informatics, vol. 14, no. 1, pp. 299-309, 2021.

[20] K. B. Bhangale and K. Mohanaprasad, "A review on speech processing using machine learning paradigm," International Journal of Speech Technology, vol. 24, no. 2, pp. 367-388, 2021.

[21] C. Wang, S. Chen, Y. Yang, F. Hu, F. Liu, and J. Wu, "Literature review on wireless sensing-Wi-Fi signal-based recognition of human activities," Tsinghua Science and Technology, vol. 23, no. 2, pp. 203-222, 2018.

[22] L. J. Zhang and D. Zhang, "Dialogic discussion as a platform for constructing knowledge: student-teachers' interaction patterns and strategies in learning to teach English," AsianPacific Journal of Second and Foreign Language Education, vol. 5, no. 1, pp. 1-24, 2020.

[23] X. Tang, S. Zhang, Y. Li, and M. Zhao, "Study on correlation of English pronunciation self-concept to English learning," English Language Teaching, vol. 6, no. 4, pp. 74-79, 2013.

[24] R. Lahaye and S. Lee, "Development of problem-based learning in an English-mediated college science course: design-based research on international instructor's four semesters instruction," Educational Technology International, vol. 19, no. 2, pp. 229-254, 2018.

[25] Y.-L. Yeh and Y.-J. Lan, "Fostering student autonomy in English learning through creations in a 3D virtual world," Educational Technology Research and Development, vol. 66, no. 3, pp. 693-708, 2018.

[26] K. A. Anwar, "Constructive teaching model in learning research concept for English language teaching students," International Education Studies, vol. 8, no. 5, pp. 62-68, 2015. 